\title{
A Multidirectional Physarum Solver for the Automated Design of Space Trajectories
}

\author{
Luca Masi \\ Mechanical \& Aerospace Department \\ University of Strathclyde \\ Glasgow, UK, G11XJ \\ Email: luca.masi@strath.ac.uk
}

\author{
Massimiliano Vasile \\ Mechanical \& Aerospace Department \\ University of Strathclyde \\ Glasgow, UK, G11XJ \\ Email: massimiliano.vasile@strath.ac.uk
}

\begin{abstract}
This paper proposes a bio-inspired algorithm to automatically generate optimal multi-gravity assist trajectories. The multi-gravity assist problem has some analogies with the better known Traveling Salesman Problem and can be addressed with similar strategies. An algorithm drawing inspiration from the Physarum slime mould is proposed to grow and explore a tree of decisions that corresponds to the possible sequences of transfers from one planet to another. Some examples show that the proposed bio-inspired algorithm can produce solutions that are better than the ones generated by humans or with Hidden Genes Genetic Algorithms.
\end{abstract}

\section{INTRODUCTION}

A gravity assist manoeuvre exploits the gravity of a planet to change the velocity of a spacecraft. As a result, substantial modifications of the orbital motion of the spacecraft can be obtained without the use of its propulsion system. An optimal sequence of gravity assist maneuvers can make challenging targets, like Saturn, accessible. The effectiveness of a sequence of gravity assist maneuvers depends on the choice of the planets (called swing-by planets in the following) and of the timing of the encounters with each planet. The problem of finding the sequence of planets and encounter dates (including resonances, or multiple encounters with the same planet) that provides the best transfer to a target object is a challenging combinatorial problem, referred to as the MGA problem, or MGAP, in this paper.

Deterministic algorithms for the solution of the MGAP are based on simplified models and an enumerative search like PAMSIT [1], or a two stage approach in which a list of possible sequences is derived from an analysis of the Tisserand's graph or from simple energetic considerations [2] and a search for optimal dates is then performed with a branch and prune type of procedure for each of the sequences.

Bio-inspired techniques for the solution of the MGAP can be found in [3], [6], [5]. In [3] the authors proposed a hybrid branch \& prune and evolutionary process that could automatically generate sequence and optimal multi-gravity assist transfer with Deep Space Maneuvers (DSM's) in a single loop. In [6] and [5] the problem is approached by using Hidden Genes Genetic Algorithms (HGGA) where each sequence is represented by a binary chromosome. The generation of an optimal solution goes through two loops: an outer loop and an inner loop. The outer loop generates the sequence of planets through a Hidden Genes Genetic Algorithm. The inner loop takes the sequence generated by the outer loop and computes an optimal set of encounter dates. Previous work by Ceriotti and Vasile [4] showed the potentiality of Ant Colony Systems at effectively solving the MGAP. The MGAP is translated into a planning and scheduling problem in which a solution is incrementally built with a modified Ant Colony Optimization algorithm.

The bio-inspired heuristic presented in this paper takes inspiration from the behaviour of a simple amoeboid organism, the Physarum Polycephalum, that is endowed by nature with simple heuristics that can solve complex discrete decision making problems. For example, it was shown that the Physarum Polycephalum is able to find the shortest path through a maze [9], recreate the Japan rail network, reproduce the designed highway network among several Mexican cities [7], solve multi-source problems with a simple geometry [8], [10], mazes [11] and transport network problems [11].

The algorithm presented in this paper is first applied to the solution of the classic Traveling Salesman Problem (TSP), and then to two instances of the MGA problem: a simple energy-based two dimensional problem without considering the actual ephemerides of the planets, and a more complex three dimensional problem with real ephemerides.

\section{Multi-Directional Discrete Decision MAKING}
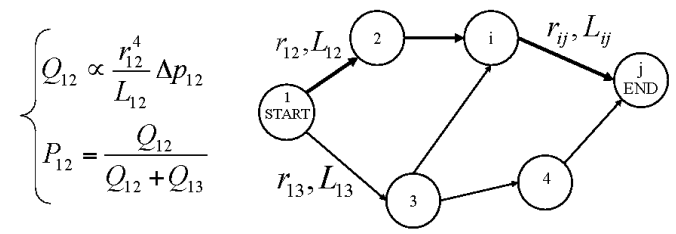

Fig. 1. Figure showing a simple graph: thicker arrows represent higher fluxes. In this example $Q_{12}>Q_{13} \Rightarrow P_{12}>P_{13}$.

The mathematical model of the Physarum's decision making process is composed of two main parts: decision network exploration and decision network growth in 


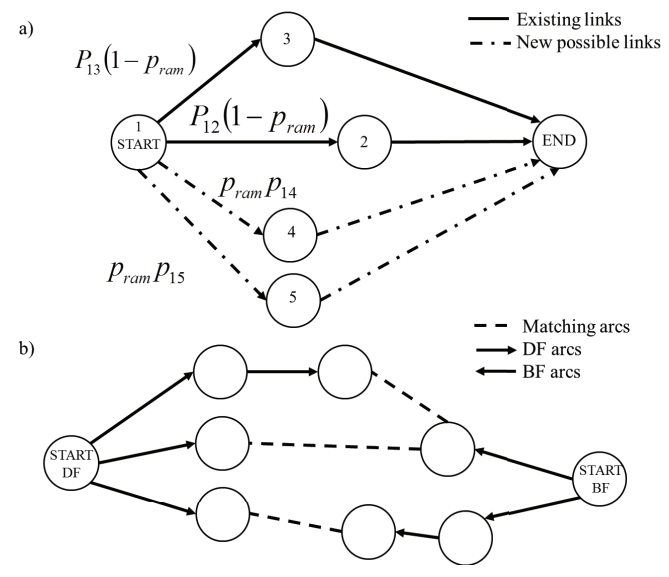

Fig. 2. Figure showing ramification towards a new node (a) and matching between decision paths in DF and BF (b).

multiple directions. They are presented in this section along with a restart procedure that mitigates the risk of stagnation. The pseudocode of the multidirectional incremental modified Physarum solver is provided in Algorithm 1.

1) Decision network exploration: The flux through the net of Physarum veins can be modelled as a classical HagenPoiseuille flow in cylindrical ducts with diameter variable with time [8], [10], [11]:

$$
Q_{i j}=\frac{\pi r_{i j}^{4}}{8 \mu} \frac{\Delta p_{i j}}{L_{i j}}
$$

where $Q_{i j}$ is the flux between $i$ and $j, \mu$ is the dynamic viscosity, $r_{i j}$ the radius, $L_{i j}$ the length and $\Delta p_{i j}$ the pressure gradient. These quantities are shown in the simple graph in Fig. 1. A variation in the diameter of the veins allows for a change in the flux. The dilation of the veins due to an increase in the flowing nutrients can be modelled using a monotonic function of the flux:

$$
\left.\frac{d}{d t} r_{i j}\right|_{\text {dilation }}=f\left(Q_{i j}\right)
$$

where $f(0)=0$, i.e., linear, sigmoidal, etc. It can be assumed that the dynamics of the veins is sufficiently slow for the flow to be considered in steady state [10] regime. The contraction of the veins, due to evaporative effects, can be assumed to be directly proportional to their radius:

$$
\left.\frac{d}{d t} r_{i j}\right|_{\text {contraction }}=-\rho r_{i j}
$$

where $\rho \in[0,1]$ is a pre-defined evaporation coefficient.

The probability associated with each vein connecting $i$ and $j$ is then computed using a simple adjacency probability matrix based on fluxes:

$$
P_{i j}= \begin{cases}\frac{Q_{i j}}{\sum_{j \in N_{i}} Q_{i j}} & \text { if } j \in N_{i} \\ 0 & \text { if } j \notin N_{i}\end{cases}
$$

where $N_{i}$ is the set of neighbouring veins to a node $i$.
TABLE I. SETTING PARAMETERS FOR THE MODIFIED PHYSARUM SOLVER

\begin{tabular}{ll}
\hline$m$ & Linear dilation coefficient, see Eq. (6). \\
$\rho$ & Evaporation coefficient, see Eq. (3). \\
$G F$ & Growth factor, see Eq. (5) \\
$N_{\text {agents }}$ & Number of virtual agents. \\
$p_{\text {ram }}$ & Probability of ramification, see Sec. II. \\
$\lambda$ & Weight on ramification, see Eq. (8). \\
\hline
\end{tabular}

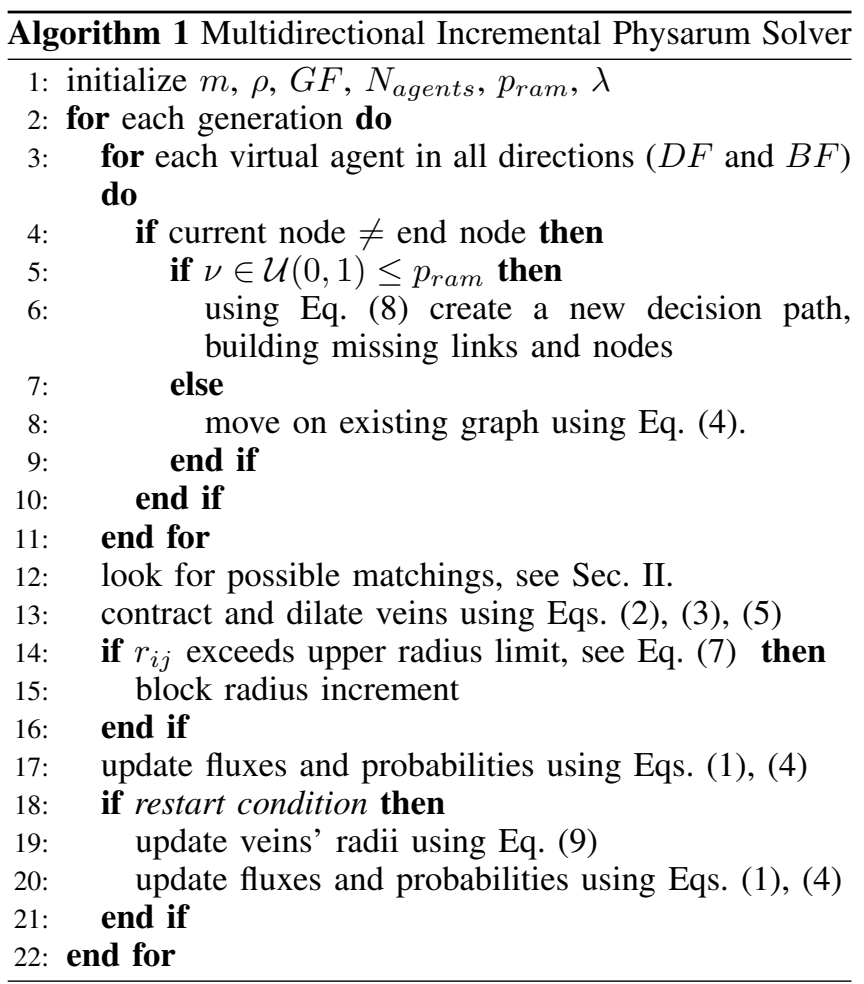

A further term in the dilation process was added in the algorithm and takes inspiration from the behaviour of the amoeba Dictyostelium discoideum. In its aggregative and slug stages, amoebae are chemotactically sensitive to a chemical known as cyclic Adenosine Monophosphate (cAMP). A starving pacemaker amoeba starts to emit cAMP, that is a call for aggregation and subsequent collective behaviour. In a computational algorithm, pacemaker can be considered the agent with best objective function. A linear dilation for the pacemaker, which is defined as the best path so far in the decision graph in terms of objective function, was here chosen:

$$
\left.\frac{d}{d t} r_{i j_{\text {best }}}\right|_{\text {elasticity }}=G F r_{i j_{\text {best }}}
$$

where $G F$ is the growth factor of the best chain of veins and $r_{i j_{\text {best }}}$ the veins' radii. This pacemaker call can be interpreted as a variable elasticity of the veins with time: best veins increase their capacity of dilation with a percentage $G F$. This is an additive term in the veins' dilation process, whose first main term is expressed in Eq. (2).

The set of Eqs. (1)-(4) can be implemented following the method proposed in [8] and resembles classical Ant Colony Optimization algorithms. Nutrients inside veins are interpreted as virtual agents that move in accord with the adjacency probability matrix in Eq. (4) on the existing graph, see line 8 of Algorithm 1. In accordance to Eq. (1), the 
flux in each vein is proportional to the fourth power of the radius and inversely proportional to the length. Once a vein is selected by a virtual agent in a generation, its radius is incremented using Eq. (2). In the present work, a function linear with respect to the product between the radius $r_{i j}^{(k)}$ of the veins traversed by agent $k$, and the inverse of the total cost of the decision taken by agent $k$, i.e., the total length $L_{t o t}^{(k)}$, will be used for the veins' dilation:

$$
\left.\frac{d}{d t} r_{i j}^{(k)}\right|_{\text {dilation }}=m \frac{r_{i j}^{(k)}}{L_{\text {tot }}^{(k)}}
$$

where the coefficient $m$ is here called linear dilation coefficient. Evaporation is taken into account using Eq. (3) for each agent. Fluxes are then calculated using Eq. (1) and probabilities are updated in accordance with Eq. (4). This mechanism of veins' diameter and flux updating corresponds to line 13 of Algorithm 1, where Eq. (5) contributes to the veins' growth of the pacemaker. An upper limit on the maximum vein radius was introduced in order to avoid veins' flux explosion and limit the converge rate. If the radius $r_{i j}$ exceeds a maximum value $r_{\max }$, the vein dilation is stopped until the radius returns again below $r_{\max }$ for the effect of evaporation. This upper limit, called $k_{\text {explosion, }}$, is given as the ratio between $r_{i j}$ and $r_{i n i}$ :

$$
k_{\text {explosion }}=\frac{r_{i j}}{r_{i n i}}
$$

where $r_{i n i}$ is the initial radius of the veins. This mechanism corresponds to lines 14 to 16 of Algorithm 1.

2) Unidirectional Growth of the Decision Network: The incremental growth of the decision network in one direction is performed in parallel by a set of virtual agents. At every node of the tree, each agent either generates a new branch or moves along an existing one. At each node, the agent has a probability $p_{\text {ram }}$ of ramification towards new nodes that are not yet linked with the current one. On line 5 of Algorithm 1, a random number $\nu$ is drawn from a uniform distribution $\mathcal{U}(0,1)$ and the condition $\nu<p_{\text {ram }}$ is verified. Assuming that the agent is at node $i$, if ramification is the choice, the agent evaluates the set of possible new branches and assigns a probability $p_{i j}$ of constructing a new link from the current node $i$ to a new possible node $j \in \bar{N}_{i}$, where $\bar{N}_{i}$ is the set of unlinked nodes (for example nodes 4 and 5 in Fig.2(a)), according to:

$$
p_{i j} \propto \frac{1}{L_{i j}^{\lambda}}
$$

where $\lambda$ is a pre-defined exponent. Fig. 2(a) shows a possible ramification from the start node: dotted lines represent feasible branches not yet existing. If an agent is at the start node it has a probability $p_{\text {ram }}$ of ramification towards the unlinked nodes 4 and 5 . If the agent decides to create a new link, a new node is selected according to Eq. (8), see line 6 of Algorithm 1.

If a set of linked nodes is available, the agent can decide, with probability $1-p_{\text {ram }}$, to traverse the existing branches in the neighborhood $N_{i}$ (see line 8 of Algorithm 1). In the case shown in Fig. 2(a) when an agent is at the start node, it can explore the already linked nodes 2 and 3. Once at node 2 or 3 the only possibility in order to complete the decision path is a new link construction between the current node and ending node.

3) Multidirectional Growth of the Decision Network: If multiple Physarum are simultaneously grown, one can explore the decision space from multiple directions. Here a bi-directional approach is presented in which two Physarum, called $D F$ and $B F$, form a network made of two superposed graphs. While growing, the two expanding Physarum have the possibility of matching decision sequences: agents can build and traverse arcs that connect nodes belonging to $D F$ and $B F$ Physarum respectively forming a single path from the heart of one Physarum to the heart of the other Physarum, see line 12 of Algorithm 1.

Figure 2(b) shows a simple case of matching between the graphs associated to two amoebae. The matched decision path is given by the union of a route in the $D F$ and one in the $B F$ through a matching arc. Several types of matching strategies were implemented and tested. The most promising strategy proceeds as follows: the best $n_{\text {seq }} \mathrm{BF}$ and DF partial routes are taken and each DF route communicates with each of the $\mathrm{BF}$ routes. The communication process randomly selects a pair of nodes along the two routes and tries to connect the two nodes with a matching arc.

In the following, the top 10 routes generated in DF and $\mathrm{BF}$ are matched assuming an equal probability of cutting any of the arcs.

4) Restart Procedure: A restart procedure was added to mitigate the risk of stagnation at local minima. If a certain condition, here called restart condition, is reached, the veins' radii are reset to:

$$
r_{i j}=r_{i n i}
$$

The restart procedure is based on the number of nodes and arcs in common between two decision sequences: after comparing all decision sequences among each other, if the minimum number of nodes in common $n_{\min }^{\text {com }}$ exceeds a threshold $n_{\text {share }}$, the algorithm is restarted. The restart procedure is summarised at lines 18 to 21 of Algorithm 1.

The main parameters of the modified Physarum solver are summarized in Table I. The initial radius of the veins $r_{i n i}$ is always set equal to 1 in the simulations presented in this paper. The complete pseudocode of the multidirectional incremental modified Physarum solver is provided in Algorithm 1. The unidirectional algorithm is a special case of the multidirectional algorithm, obtained by freezing the $B F$, i.e., flux and graph growth are allowed in only one direction.

\section{APPLiCATION TO THE TSP}

The TSP, a classical problem in combinatorial optimization, was used as benchmark for the performance evaluation of the modified unidirectional and multidirectional Physarum algorithms. Given a set $N_{\text {cities }}$ cities whose reciprocal distance $l$ is known, the TSP is the problem of finding the shortest tour that visits each city exactly once. TSPLIB [16] was used to benchmark the proposed Physarum algorithm on the TSP problem. In particular, Fig. 3 shows a comparison between the multidirectional and the unidirectional modified Physarum solver for the TSP test cases Eil51 from TSPLIB, featuring 51 cities. Setting values are 


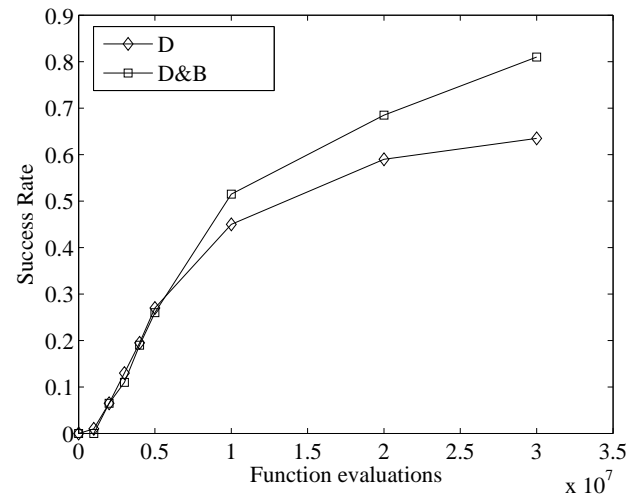

Fig. 3. Variation of the success rate with the number of function evaluations - Eil51 TSP test case.

$m=5 \times 10^{-3}, \rho=10^{-4}, G F=5 \times 10^{-3}, N_{\text {agents }}=100$, $p_{\text {ram }}=1, \lambda=0$. The value $k_{\text {explosion }}=5$, see Eq. (7) and restart with $n_{\text {share }}=\frac{\text { dim }}{2}$ were selected, where $\mathrm{dim}$ is the number of cities. Fig. 3 reports the success rate (i.e., more runs successfully converge to the known best solution) over 200 independent runs and show that the multidirectional modified Physarum algorithm with matching ability (D\&B) provides higher success rate than the unidirectional modified Physarum algorithm (D) when applied to TSP problems. In particular for Eil51 the gain using D\&B instead of D reaches approximately $20 \%$ at $3 \times 10^{7}$ function evaluations. It should be noted that there is an initial transient phase where the performance of the multidirectional solver do not exceed the performance of the unidirectional solver because the two expanding Physarum cause an initial increase in the number of function evaluations while the growth of the veins is still low. After this initial transient, the two Physarum start to reduce the search space and the role of communication becomes crucial.

\section{A. Comparison with Other Algorithms}

Although the idea of this paper is to show that multidirectionality increases the performance of the Physarum algorithm, a comparison with known and tested algorithms is proposed in the following. Note that the Physarum algorithm does not contain either tailored heuristics for TSP or local optimization heuristics, like 2-opt [18], or static or dynamic candidate lists [15], [14]. For this comparative test the Physarum was applied to the solution of problems Eil51, Eil76, Eil101, Kroa100 and Rat195 of the TSBLIB.

1) ACS, MMAS, KniesG, KniesL, SA for TSP.: For this set of comparative tests, mean, best and standard deviation are used as performance indicators, see Table II and Table III, in order to have a fair comparison with the results in the literature. Values in Table II for the ACO-inspired algorithms are from [14], [15]. ACS is an implementation of Ant Colony System, while MMAS is a Max-Min Ant System algorithm, which is considered the state of the art of ACO-inspired algorithms. Table II shows that the proposed Physarum algorithm is comparable to state of the art ACS algorithms. The values in Table III are from [17]. KniesG refers to Kohonen Network Incorporating Explicit Statistics Global [12], KniesL to its local version [12], while SA
TABLE II. TSP BENCHMARK USED IN [14] WITH MEAN VALUE, STANDARD DEVIATION AND BEST VALUE AT $3 \times 10^{7}$ FUNCTION CALLS)

\begin{tabular}{lccc}
\hline & $\begin{array}{c}\text { Physarum } \\
\text { D\&B }\end{array}$ & MMAS & ACS \\
\hline \hline Eil51 & & & \\
mean & 426.8 & 427.2 & 428.1 \\
variance & 0.92 & 1.13 & 2.48 \\
best & 426 & 426 & 426 \\
Kroa100 & & & \\
mean & 21352.9 & 21352.1 & 21420.0 \\
variance & 94.7 & 50.3 & 141.7 \\
best & 21282 & 21282 & 21282 \\
\hline
\end{tabular}

ACS is from [14], 15 runs performed. MMAS is from [15], 25 runs performed. Physarum, 25 runs performed for Kroa100, 200 for Eil51.

TABLE III. TSP BENCHMARK FROM [17], MEAN VALUE AT $10^{7}$ FUNCTION CALLS

\begin{tabular}{lcccc}
\hline & $\begin{array}{c}\text { Physarum } \\
\text { D\&B }\end{array}$ & KniesG & KniesL & SA \\
\hline \hline & & & & \\
Eil51 & 427.8 & 438.2 & 438.2 & 435.9 \\
Eil76 & 543.7 & 567.5 & 564.8 & 567.8 \\
Eil101 & 649.8 & 664.4 & 658.3 & 665.1 \\
Rat195 & 2397.0 & 2599.9 & 26073.0 & 2631.7 \\
\hline
\end{tabular}

KniesG, KniesL, SA are from [17], runs performed n/a. Physarum, 200 runs performed.

indicates Simulated Annealing [13]. The results in this table demonstrate the superior ability of the Physarum algorithm at finding good solutions for the test cases Eil51, Eil76, Eillo1 and Rat195.

\section{MGAP 2D ENERGY MODEL}

The MGAP is here formulated as a simple planar trajectory model. The motion of planets and the spacecraft is assumed to occur in a plane. The orbits of all the planets are assumed to be circular. The swing-by of a planet is assumed to produce an instantaneous variation of the velocity of the spacecraft with respect to the Sun. The variation of the velocity is only due to the gravity of the planet and no propelled maneuvers are considered in this model (see [1] for more details).

Given a sequence of planetary encounters $\{A, B, \ldots\}$, the modulus $v_{\infty, L}$ and the direction $\beta$ of the velocity vector of the spacecraft with respect to the velocity $V_{p}$ of planet A at departure, the spacecraft follows an orbit with, at most, two intersections with the orbit of the following planet in the sequence (see Fig. 4). With reference to Fig. 4, if Planet $\mathrm{A}$ is the Earth and the launch happens at 4, then, if no resonances are considered, at most two possible transfer arcs are possible: $4-1$ and $4-2$. This is true for each transfer between two planets. Note that planets are assumed to always be at the intersection points thus no actual phasing is considered.

For each intersection, two different swing-by's are possible with two outgoing velocities. Hence each planet-toplanet sequence leads to four possible outgoing velocities and therefore four possible outgoing branches. Resonances are taken into account by introducing a further discrete parameter that defines the number of complete revolutions around the Sun (limited to 2 in the following). Each intersection, including resonances, represents a node in the tree 

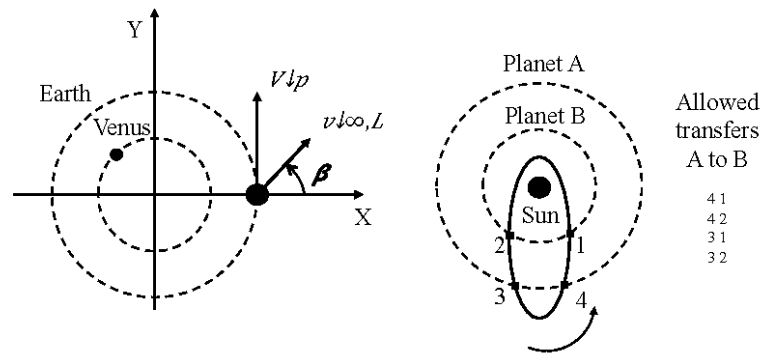

Fig. 4. Initial conditions and different transfers on the same orbit

TABLE IV. Success Rate: Physarum, Physarum D\&B. EARTH TO JUPITER TRANSFERS: EJ7 $\left(v_{\infty, L}=7 \mathrm{~km} / \mathrm{s}, \alpha=1.5 \pi\right)$, EJ5 $\left(v_{\infty, L}=5 \mathrm{~km} / \mathrm{s}, \alpha=1.5 \pi\right)$

\begin{tabular}{|c|c|c|}
\hline \multicolumn{3}{|c|}{ Success Rate } \\
\hline Instance & EJ5 & EJ7 \\
\hline Func. eval. & 5000,30000 & 5000,30000 \\
\hline Physarum & 0.66 & 0.81 \\
\hline Physarum D\&B & 0.45 & 0.94 \\
\hline & Relative Simulation & Time \\
\hline Instance & EJ5 & EJ7 \\
\hline Func. eval. & 30000 & 30000 \\
\hline Physarum & 1 & 1 \\
\hline Physarum D\&B & 0.74 & 0.68 \\
\hline
\end{tabular}

in Fig. 2. For the solution of this particular instance of the MGAP, the decision tree is built only forward while it is explored both forward and backward. The matching process links complete paths explored backwards to partial paths generated and explored forward.

The cost of each transfer from intersection $i$ to intersection $j$ is the time of flight $T o F_{i j}$ and the total objective function, to be minimised, is the total time of flight $T o F_{t o t}$. The Physarum algorithm is applied by replacing $L_{\text {tot }}^{(k)}$ in Eq. (1) and $L_{i j}$ in Eq. (6) respectively with $T o F_{t o t}^{(k)}$ and $T o F_{i j}$. The length of the sequence of planets is bounded from above with an upper limit of 4 consecutive swing-by's.

The Physarum and the Multidirectional Physarum solvers were tested on two instances of the Earth to Jupiter transfer problems, named respectively EJ5 and EJ7. The EJ5 transfer has a departure velocity $v_{\infty, L}=5 \mathrm{~km} / \mathrm{s}$ with a departure

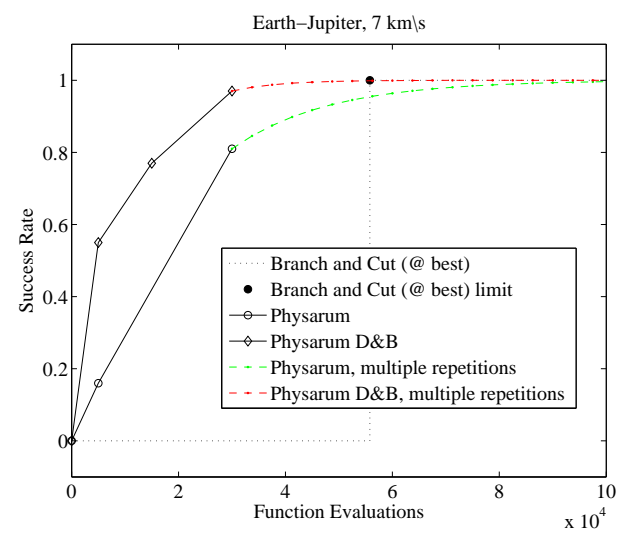

Fig. 5. Success rate: Physarum, Physarum D\&B and Branch \& Cut. Earth to Jupiter transfer EJ7, $v_{\infty, L}=7 \mathrm{~km} / \mathrm{s}, \alpha=1.5 \pi$. angle $\beta=1.5 \pi$, while the EJ7 has a departure velocity $v_{\infty, L}=7 \mathrm{~km} / \mathrm{s}$ and a departure angle $\beta=1.5 \pi$. The pericentre altitude for the swing-by's of Mercury, Venus, Earth, Mars and Pluto is $200 \mathrm{~km}$, while it is 5 Jovian radii for Jupiter, 2 planetary radii for Saturn, and one planetary radius for Uranus and Neptune.

The parameters of the Physarum solver, for this and the following test case, were set as follows: $m=0.005$, $\rho=0.0001, G F=0.005, N_{\text {agents }}=20, p_{\text {ram }}=0.6$, $\lambda=0, k_{\text {explosion }}=5, Q_{\max }=30$. For this and all the cases in the remainder of this paper $n_{\text {share }}=\infty$. Both the unidirectional and multidirectional algorithms were run for 200 times on each of the two problems for a number of function evaluations that ranged between 5000 and 100000 . It is important to recall that one function evaluation is the evaluation of a single arc connecting two nodes in the search tree in Fig. 2.

The best solution found by both Physarum solvers is the sequence of planets EVVEMJ (Earth, Venus, Venus, Earth, Mars, Jupiter) both for EJ7 and EJ5 with cost 1.48 and 1.38 years, and is in line with the results obtained with the exhaustive search presented in [1].

Table IV shows that the success rate for the multidirectional solvers is $10 \%$ higher, at 30000 function evaluations, and more than $20 \%$ higher, at 5000 function evaluations, than the unidirectional solver. Furthermore, the Physarum D\&B algorithm is around $30 \%$ faster, thanks to the matching approach that rapidly combines and discovers optimal sequences.

Fig. 5 shows the success rate for the unidirectional, multidirectional and a deterministic branch \& cut algorithm. The branch \& cut algorithm builds the tree of sequences in a manner similar to the unidirectional solver but cuts branches when the partial value of the objective function is already above the best value of the objective function for a complete transfer. Fig. 5 shows that the success rate of the branch \& cut algorithm is zero up to a minimum number of function evaluation after which it jumps to 1 . The switching point from 0 to 1 is reached at around 55000 function evaluations while the Physarum algorithm finds good solutions for any number of function evaluations, with an $93 \%$ success at 30000 function evaluations.

The figures show also the extrapolated success rate of the two algorithms assuming that the algorithm is run multiple times for a constant number of function evaluations. The extrapolation is computed by considering the probability of success that the algorithm would have if it was launched multiple times for 30000 function evaluations each time. The probability of success is $1-p_{30}^{n}$, where the probability of failure at 30000 function evaluations is $p_{30}$ and $n$, in this case, is the number of repetitions.

Note that, if each planet-encounter time pair was a city in a TSP, each planet could be revisited $K$ times and $N_{P}$ planets were available per each encounter, then the maximum length of a sequence would be $K N_{P}$ and the number of alternative complete trajectories would be $\left(K N_{P}\right)^{\left(4 K N_{P}\right)}$. 


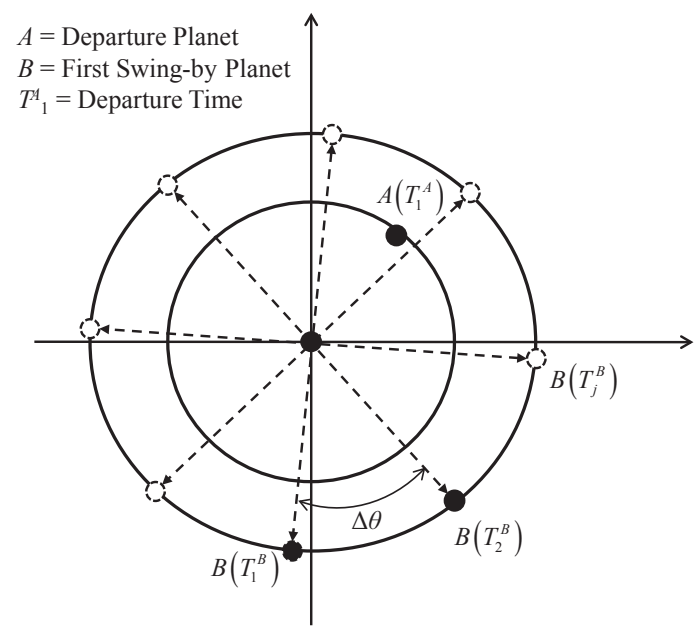

Fig. 6. Formulation in position for transfers between two planets.

\section{MGAP 3D LAMBert MOdel}

In this section, the MGAP is modelled using the actual ephemerides of the planets. Given two planets, $A$ and $B$, and the dates $T_{i}^{A}$ and $T_{j}^{B}$ at which the spacecraft is at planet $A$ and $B$, the solution of the Lambert's problem gives the conic arc connecting the two planets and the associated velocity vectors at the beginning and at the end of the arc. If $B$ is a swing-by planet and $C$ is the following planet in a sequence, the mismatch of velocity at $B$ between the velocity vector at the end of the $A-B$ arc (incoming velocity) and the velocity vector at the beginning of the $B-C$ arc (outgoing velocity) is partially compensated by assuming that the gravity of the planet $B$ is steering the incoming velocity. Since the altitude at which the spacecraft is allowed to swing-by the planet is limited and the mass of the planet is given, not all incoming velocities can be naturally rotated to match the outgoing velocities (i.e., the velocity difference between the velocity vector at the beginning of the arc following the swing-by and the velocity of the planet). In this case a propelled maneuver $\Delta v_{i}$ is placed at the pericentre of the swingby hyperbola. This combination of powered maneuver and gravity steering is called powered gravity assist manoeuvre or powered swing-by.

2) Formulation in Position: The position and velocity of the first planet in a sequence, say $A$, are calculated from the ephemerides for the given vector of discrete times $\mathbf{T}^{A}=$ $\left[T_{1}^{A}, T_{2}^{A}, \ldots, T_{i}^{A}, \ldots\right]^{T}$. For all the subsequent planets, up the last one in the sequence, instead, the times are derived from the phase angles (see Fig. 6). Assuming $B$ is the next planet in the sequence, following $A$, and $\theta_{1}^{B}$ is the phase angle of $B$ on its orbit at time $T_{1}^{A}$, the position, velocity and time $T_{j}^{B}$ of $B$ are computed for $\theta_{j}^{B}=\theta_{1}^{B}+\Delta \theta_{j}$, with $j=1, \ldots, n_{B}$ and $n_{B}=2 \pi / \Delta \theta_{j}$. The time corresponding to a given discrete phase angle can be computed from the time equation in the form $T_{j}^{B}=f\left(\theta_{j}^{B}+2 k_{r} \pi\right)$. Note that, this model is applied also in reverse from the last planet to the first. In this case the position and velocity of the last planet are calculated from the ephemerides given a time vector that spans the desired arrival temporal window.

\section{A. Generation of the Search Tree}

If the vectors of encounter dates for planet $A$ and $B$ are respectively $\mathbf{T}^{A}=\left[T_{1}^{A}, T_{2}^{A}, \ldots, T_{i}^{A}\right]^{T}$ and $\mathbf{T}^{B}=$ $\left[T_{1}^{B}, T_{2}^{B}, \ldots, T_{j}^{B}\right]^{T}$, then the set of possible transfers from $A$ to $B$ can be represented with a matrix where each element $z_{i j}^{A B}$ of the matrix is the cost associated with a particular $T_{i}^{A} \rightarrow T_{j}^{B}$ transfer. If multiple alternative planets are available the matrix becomes three dimensional, with the third dimension containing all possible planets. Note that each element of the matrix is a node in the tree of decisions that the Physarum incrementally builds, therefore only the nodes that the Physarum explores are actually generated and added to the tree. However, when a planet $A$ the Physarum generates a new branch towards $B$ all the transfers to $B$ are evaluated and one selected to be added as a new node to the tree, the other transfers are stored for later addition of other nodes.

The cost $z_{i j}^{A B}$ is the launch excess velocity $\Delta v_{0}$ if planet $A$ is the departure planet, the powered swing-by cost $\Delta v_{i}$ if $A$ is a swing-by planet, or the sum of $\Delta v_{i}$ and the arrival excess velocity $\Delta v_{f}$ if $B$ is the final planet. The cost of a complete transfer is then the sum of the departure $\Delta v_{0}$ plus all the $\Delta v_{i}$ for all the planetary encounters and $\Delta v_{f}$. In the Physarum algorithm, the variables $L_{t o t}^{(k)}$ in Eq. (1) and $L_{i j}$ in Eq. (6) are then replaced by, respectively, $\Delta v_{t o t}^{(k)}$ and $z_{i j}^{A B}$.

From a given planet at a particular node, a new planet is selected with a probability proportional to the inverse of the difference of the semimajor axis of the new planet with respect to the current one. Once the costs for the whole vector $\mathbf{T}^{B}$ is available, a transfer is selected, for example $T_{2}^{A} \rightarrow T_{2}^{B}$, with Eq. (4), where only the costs $z_{i j}^{A B}$ are used to compute the fluxes. If $\nu \in \mathcal{U}(0,1)>p_{\text {ram }}$, the algorithm does not evaluate the cost for a new set of transfer arcs (i.e., does not build a new branch) but selects an existing arc among the available possibilities using Eq. (4). The process is repeated until the final target planet is reached and a complete decision sequence is built. If, during the construction of a solution, no transfer arcs can be found that satisfy the constraints, then the construction is terminated and an infinite cost (or equivalently a zero probability) is associated to the resulting partial solution. Eq. (6) was slightly modified by substituting $L_{\text {tot }}^{(k)}$ with $L_{t o t}^{(k)}+1$ in order to avoid possible singularities that may appear with the MGA model.

1) Local Solution Improvement Strategy: In order to improve the quality of the solutions, a local search procedure inspired to the 2-opt local search strategy was added to the algorithm. If $\mathbf{s}=\left[A, T_{2}^{A}, B, T_{7}^{B}, C, T_{12}^{C}, D, T_{16}^{D}\right]^{T}$ is a solution vector, the local improvement checks whether a positive or negative increment of $T_{2}^{A}, \delta T$, improves the solution. If, for example, $\Delta v_{t o t}\left(A, T_{2}^{A}, B, T_{7}^{B}, C, T_{12}^{C}, D, T_{16}^{D}\right)>$ $\Delta v_{\text {tot }}\left(A, T_{2}^{A}+\delta T, B, T_{7}^{B}, C, T_{12}^{C}, D, T_{16}^{D}\right)$, then $T_{2}^{A}$ is replaced by $T_{2}^{A}+\delta T$. The same $\delta T$ is repeatedly added (or subtracted) to $T_{2}^{A}$ till no improvement is registered. The process is then applied to $T_{7}^{B}$ and the other dates till $T_{16}^{D}$, and repeated backwards from $T_{16}^{D}$ to $T_{2}^{A}$. Note that the modified dates do not necessarily correspond to the discretised phase angles. 
2) Algorithm and problem settings: Along with the algorithm's parameters $m, \rho, G F, N_{\text {agents }}, p_{\text {ram }}, r_{i n i}$, $k_{\text {explosion }}$ and $\lambda$ introduced in Sec. II, a number of additional quantities needs to be defined to characterize a particular instance of the MGA problem. In particular, the departure planet $P_{0}$, the upper and lower boundaries on the swing-by altitude divided by the radius of the planet $h_{\text {low }}$ and $h_{u p}$, the set of available swing-by planets $P_{s}=\left\{P_{1}, P_{2}, \ldots, P_{N_{P}}\right\}$, maximum number of swing-by's $n_{s_{\max }}$, maximum number of resonances $r e s_{\max }$, interval of dates defining the launch window $T_{\text {launch }}$, the interval of dates defining the arrival window $T_{\text {arrival }}$, the upper and lower boundaries on the time of flight $T o F_{i j}^{l o w}$ and $T o F_{i j}^{u p}$ for each leg connecting two planets $i$ and $j$, the final target planet $P_{\text {target }}$, the grid spacing in angle $\Delta \theta_{i j}$ and the upper and lower boundaries on launch and arrival velocities, respectively $\Delta v_{0}^{\max }, \Delta v_{0}^{\min }$ and $\Delta v_{f}^{\max }, \Delta v_{f}^{\min }$. The settings of the algorithm for the cases in this and following section can be found in Tables V, VI. Planets are identified with the following letters Me(Mercury), V(Venus), E(Earth), M(Mars), J(Jupiter), $\mathrm{S}$ (Saturn), U(Uranus), N(Neptune), P(Pluto). For example the sequence EVVEJS means Earth-Venus-Venus-EarthJupiter-Saturn. Parameters $m, \rho, G F, N_{\text {agents }}, p_{\text {ram }}$ are set with the same values used for the TSP and MGA 2D energy model examples (Sec. III and Sec. IV), whilst $r_{i n i}$ is increased from 1 to 2 , and $k_{\text {explosion }}$ consequently, in order to have a maximum radius of 5 .

Note that, if, in this case, each planet-encounter time pair was a city in a TSP, each planet could be revisited $K$ times, $N_{P}$ planets were available per each encounter and each pair of planets required the evaluation of $\left(N_{P} K N_{T}\right)^{2}$ transfer arcs, where $N_{T}$ is the number of discrete encounter dates, then the total number of transfers to be evaluated would be $K N_{P}\left(N_{P} K N_{T}\right)^{2}$. If the transfer arcs were put together in a sequence, the number of alternative sequences would be $\left(K N_{P}\right)^{\left(N_{P} K N_{T}\right)}$.

\section{B. Case Study: Mission to Saturn}

This test case reproduces the Cassini mission, launched in November 1997 to Saturn [19]. For this particular example the maximum number of swing-by's is 4 and the swingby planet can be selected from a set of 4 planets with a maximum of 2 repeating planets in the same sequence. The code was implemented in Matlab ${ }^{\circledR}$ R2010b. The simulation lasted for approximately 7 hours on an Intel Core (TM) i5$25003.30 \mathrm{GHz}$. Table VII shows the top solutions for the sequences found by the Physarum: EVVEJS with a cost of $10.0873 \mathrm{~km} / \mathrm{s}$, EVJS with a cost of $10.5125 \mathrm{~km} / \mathrm{s}$ and EVVJS with a cost of 11.6184 .

Table VIII shows a comparison between the best solution found by the Physarum solver working in only one direction versus, the best solution found by the Physarum solver with multidirectional matching ability and the solution found by the Hidden Genes solver proposed in [5]. This figure shows that the Physarum D\&B is able to find the best solution. Not only does the multidirectional Physarum yield the best results but it also provides a more even spreading of the $\Delta v_{i}$ maneuvers. In fact, the solutions found by incrementally building the sequences in only one direction (from Earth to Saturn) tend to have low costs at departure and first swing-by, with a higher cost at the second swingby (approximately $2.6 \mathrm{~km} / \mathrm{s}$ in the example in Table VIII); this is intrinsically due to the fact that any forward search algorithm (including deterministic branch and prune ones) is biased by the heuristic that is used to locally generate and select new branches. The parallel backward and forward search with intermediate matching completely removes this bias.

TABLE V. PROBLEM DEFINITION PARAMETERS

\begin{tabular}{ll}
\hline Parameter & Value \\
\hline Set of Available Planets, $P_{s}$ & $\{$ V E J S $\}$ \\
$n_{s_{\max }}$ & 4 \\
res $_{\max }$ & 1 \\
$T_{\text {launch }}$ & November-December 1997 \\
$T_{\text {arrival }}$ & July-December 2007 \\
{$\left[\Delta v_{0}^{\min }, \Delta v_{0}^{\max }\right]$} & {$[3,5] \mathrm{km} / \mathrm{s}$} \\
{$\left[\Delta v_{f}^{\text {min }}, \Delta v_{f}^{\max }\right]$} & {$[0,8] \mathrm{km} / \mathrm{s}$} \\
$h_{\text {low }}$ for $\{\mathrm{E} \mathrm{V} \mathrm{J}\}$ & {$[0.050 .050 .1]$} \\
$h_{\text {up }}$ for $\{\mathrm{E} \mathrm{V} \mathrm{J}\}$ & {$[101080]$} \\
\hline
\end{tabular}

TABLE VI. LOWER AND UPPER BOUNDARIES ON THE TIME OF FLIGHT AND $\Delta \theta$

\begin{tabular}{cccccccc}
\hline & $\mathrm{Me}$ & $\mathrm{V}$ & $\mathrm{E}$ & $\mathrm{M}$ & $\mathrm{J}$ & $\mathrm{S}$ & \\
\hline $\mathrm{lb}$ [day] & 0 & 0 & 300 & 0 & 0 & 0 & $\mathrm{Me}$ \\
$\mathrm{ub}$ [day] & 0 & 0 & 1000 & 0 & 0 & 0 & $\mathrm{Me}$ \\
$\Delta \theta$ [deg] & 0 & 0 & 4 & 0 & 0 & 0 & $\mathrm{Me}$ \\
$\mathrm{lb}$ [day] & 0 & 100 & 30 & 300 & 500 & 0 & $\mathrm{~V}$ \\
$\mathrm{ub}$ [day] & 0 & 500 & 500 & 2000 & 3000 & 0 & $\mathrm{~V}$ \\
$\Delta \theta$ [deg] & 0 & 2 & 1 & 2 & 0.5 & 0 & $\mathrm{~V}$ \\
$\mathrm{lb}$ [day] & 300 & 100 & 350 & 100 & 800 & 0 & $\mathrm{E}$ \\
$\mathrm{ub}$ [day] & 1000 & 200 & 745 & 600 & 1500 & 0 & $\mathrm{E}$ \\
$\Delta \theta$ [deg] & 2 & 1.6 & 1 & 2 & 0.4 & 0 & $\mathrm{E}$ \\
$\mathrm{lb}$ [day] & 0 & 0 & 0 & 0 & 0 & 0 & $\mathrm{M}$ \\
$\mathrm{ub}$ [day] & 0 & 0 & 0 & 0 & 0 & 0 & $\mathrm{M}$ \\
$\Delta \theta$ [deg] & 0 & 0 & 2 & 2 & 0.5 & 0.52 & $\mathrm{M}$ \\
$\mathrm{lb}$ [day] & 0 & 0 & 0 & 0 & 0 & 1500 & $\mathrm{~J}$ \\
$\mathrm{ub}$ [day] & 0 & 0 & 0 & 0 & 0 & 2900 & $\mathrm{~J}$ \\
$\Delta \theta$ [deg] & 0 & 0 & 0 & 0 & 0 & 0.2 & $\mathrm{~J}$ \\
\hline
\end{tabular}

\section{CONCLUSIONS}

This paper introduced a novel bio-inspired method for single objective discrete optimization that can effectively solve MGA trajectory problems. The algorithm was first applied to the TSP showing good performance if compared with ACS and other TSP specific algorithms. Furthermore, it was demonstrated that a multidirectional search is more efficient than a unidirectional search, when applied to the solution of reversible decision-making problems. The algorithm was then applied to two instances of the MGAP. In the first case the proposed algorithm showed the ability to quickly find optimal solutions for two variants of an Earth to Jupiter transfer, outperforming a classical unidirectional branch \& cut technique.

In the second case, the algorithm was applied to the solution of an Earth to Saturn transfer with powered swingby's and compared against a Hidden Genes solver. In this case, the multidirectional Physarum was able to find the best result providing a more even spreading of the $\Delta v$ maneuvers. 
TABLE VII. BEST THREE SEQUENCES FOR THE CASSINI TEST CASE AT 40000 FUNCTION EVALUATIONS

\begin{tabular}{lcl}
\hline Sequence & Cost $[\mathrm{km} / \mathrm{s}]$ & Epoch [MJD2000] \\
\hline EVVEJS & 10.087 & {$[-779.160,-595.763,-181.432,-132.692,463.099,2737.50]$} \\
EVJS & 10.512 & {$[-783.0,-636.054,296.144,2765.595]$} \\
EVVJS & 11.618 & {$[-766.960,-585.780,-164.193,483.760,2895.944]$} \\
\hline
\end{tabular}

TABLE VIII. CASSINI TEST CASE: COMPARISON BETWEEN UNIDIRECTIONAL, MULTI-DIRECTIONAL Physarum (BOLD) AND HGGA (ITALICS)

\begin{tabular}{ccccccc}
\hline & $\mathrm{E}$ & $\mathrm{V}$ & $\mathrm{V}$ & $\mathrm{E}$ & $\mathrm{J}$ & $\mathrm{S}$ \\
\hline & $5 / 11 / 1997$ & $2 / 4 / 1998$ & $25 / 6 / 1999$ & $20 / 8 / 1999$ & $17 / 4 / 2001$ & $25 / 10 / 2007$ \\
Date & $\mathbf{1 3 / 1 1 / 1 9 9 7}$ & $\mathbf{1 5 / 5 / 1 9 9 8}$ & $\mathbf{4} / 7 / \mathbf{1 9 9 9}$ & $\mathbf{2 1 / 8 / 1 9 9 9}$ & $\mathbf{8 / 4 / 2 0 0 1}$ & $\mathbf{1 / 7 / 2 0 0 7}$ \\
& $30 / 11 / 1997$ & $20 / 5 / 1998$ & $26 / 6 / 1999$ & $19 / 8 / 1999$ & $24 / 3 / 2001$ & $1 / 1 / 2007$ \\
\hline & & 148.3 & 448.5 & 55.7 & 606.8 & 2382.0 \\
$T o F$ & & $\mathbf{1 8 3 . 4}$ & $\mathbf{4 1 4 . 3}$ & $\mathbf{4 8 . 7}$ & $\mathbf{5 9 5 . 8}$ & $\mathbf{2 2 7 4 . 4}$ \\
{$[d a y]$} & & 171.5 & 402.0 & 53.8 & 582.6 & 2120.5 \\
\hline Pericentre & & 2554.8 & 19597.3 & 1609.3 & 4480675.7 & \\
Altitude & & $\mathbf{1 0 4 8 4 . 0}$ & $\mathbf{8 7 2 . 2}$ & $\mathbf{2 8 3 2 . 4}$ & $\mathbf{4 6 3 8 4 5 4 . 8}$ & \\
{$[\mathrm{km}]$} & 27471.0 & 605.3 & 1810.7 & 5167772.8 & \\
\hline & & 0.2944 & 2.5265 & $3.3 \times 10^{-4}$ & $3.4 \times 10^{-3}$ & 4.2009 \\
$\Delta v$ & 3.2893 & $\mathbf{3 . 9 5 0 2}$ & $\mathbf{0 . 9 3 0 9}$ & $\mathbf{9 . 0} \times \mathbf{1 0}^{-4}$ & $\mathbf{5 . 6} \times \mathbf{1 0}^{-\mathbf{4}}$ & $\mathbf{4 . 2 2 9 8}$ \\
{$[\mathrm{km} / \mathrm{s}]$} & 3.7790 & 2.6330 & $1.1 \times 10^{-5}$ & $1.4 \times 10^{-6}$ & $1.9 \times 10^{-4}$ & 4.2730 \\
\hline \hline Total & & & & & & 10.3150 \\
$\Delta v$ & & & & & & 10.6873 \\
{$[\mathrm{~km} / \mathrm{s}]$} & & & & & &
\end{tabular}

\section{REFERENCES}

[1] S Pessina, S Campagnola, M Vasile. Preliminary Analysis of Interplanetary Trajectories with Aerogravity and Gravity Assist Manoeuvres. 54th International Astronautical Congress, Bremen, Germany, 2003.

[2] A.E. Petropoulos, J.M Longuski, E.P. Bonfiglio. Trajectories to Jupiter via Gravity Assist from Venus, Earth and Mars. Journal of Spacecraft and Rockets, 37(6):776-783, 2000.

[3] M Vasile, P. De Pascale. On the Preliminary Design of Multiple Gravity-Assist Trajectories. Journal of Spacecraft and Rockets, 42(4): 794-805, 2006.

[4] M Ceriotti, M Vasile. Automated MGA Trajectory Planning with an ACO-inspired Algorithm. Acta Astronautica, 67(910): 1202-1217, 2010.

[5] A Gad, O Abdelkhalik. Hidden Genes Genetic Algorithm for MultiGravity-Assist Trajectory optimization. Journal of Spacecraft and Rockets, 48(4): 629-641, 2011.

[6] J.A Englander, B.A Conway, T Williams. Automated Interplanetary Trajectories Planning. AIAA/AAS Astrodynamics Specialist Conference, Minneapolis, Minnesota, 2012.

[7] A Adamatzky, G.J Martinez, S.V Chapa-Vergara, R AsomozaPalacio, C.R Stephens. Approximating Mexican Highways with Slime Mould. Natural Computing, 10(3): 1195-1214, 2011.

[8] D.S Hickey, L.A Noriega. Insights into Information Processing by the Single Cell Slime Mold Physarum Polycephalum. UKACC Control Conference, , Manchester, UK, 2008.

[9] T Nakagaki, H Yamada, A Toth. Maze-Solving by an Amoeboid Organism. Nature, 407(6803): 470, 2000.

[10] A Tero, K Yumiki, R Kobayashi, T Saigusa, T Nakagaki. FlowNetwork Adaptation in Physarum Amoebae. Theory in Biosciences, 127(2): 89-94, 2008

[11] A Tero, R Kobayashi, T Nakagaki. Physarum Solver: a Biologically Inspired Method of Road-Network Navigation. Physica: A Statistical Mechanics and its Applications, 363(1): 115-119, 2006.

[12] N Aras, BJ Oommen, IK Altinel. The Kohonen Network Incorporating Explicit Statistics and its Application to the Traveling Salesman Problem. Neural Networks, 12(9): 12731284, 1999.

[13] M Budinich. A Self-organizing Neural Network for the Traveling Salesman Problem that is Competitive with Simulated Annealing. Neural Computation, 8: 416424, 1996.

[14] M Dorigo, LM Gambardella. Solving Symmetric and Asymmetric TSPs by Ant Colonies. Proceedings of IEEE International Conference on Evolutionary Computation,622-627, 1996.

[15] T Stuetzle, H Hoos. Max-Min Ant System and Local Search for the Traveling Salesman Problem. IEEE International Conference on Evolutionary Computation, 309-314, 1997.
[16] TSPLIB, library of instances for travelling salesman and vehicle routing problems, Ruprecht Karls Universitaet Heidelberg, URL: http://comopt.ifi.uni-heidelberg.de/software/TSPLIB95/.

[17] X. Zhang, L. Tang. A New Hybrid Ant Colony optimization Algorithm for the Traveling Salesman Problem. Advanced Intelligent Computing Theories and Applications: With Aspects of Artificial Intelligence, 148-155, 2008.

[18] Zar Chi Su Su Hlaing, May Aye Khine. Solving Traveling Salesman Problem by Using Improved Ant Colony Optimization Algorithm. International Journal of Information and Education, 1(5): 404-409, 2011.

[19] DL Matson, LJ Spilker, JP Lebreton. The Cassini Huygens Mission to the Saturnian System. Space Science Reviews, 104(1-4): 1-58, 2002. 